\title{
ON A WEDDERBURN PRINCIPAL THEOREM FOR THE FLEXIBLE ALGEBRAS(1)
}

\author{
BY
}

\author{
ROBERT A. CHAFFER
}

ABSTRACT. A strictly power-associative algebra $A$ over a field $K$ is said to have a Wedderburn decomposition if there is a subalgebra $S$ of $A$ such that $A=S+N$, where $N$ is the nil radical of $A$, and $S=A-N$. A Wedderburn principal theorem for a class of algebras is a theorem which asserts that the algebras $A$, in the class, with $A-N$ separable have Wedderburn decompositions. It is known that there is no such theorem for the class of noncommutative Jordan algebras. A partial result in this direction is the following theorem.

Theorem. Let $A$ be a strictly power-associative, flexible algebra over a field $F$ with characteristic not 2 or 3, with $A-N$ separable and such that $A=A_{1} \oplus A_{2} \oplus \ldots \oplus A_{n}$ where each $A_{i}$ has $A_{i}-N_{i}$ simple and has more than two pairwise orthogonal idempotents. Then $A=S+N$ where $S$ is a subalgebra of $A$.

It is known from several examples [11, p. 147] and [14, p. 179] that the noncommutative Jordan algebras, as a class, do not satisfy the Wedderburn principal theorem. That is, there exist noncommutative Jordan algebras $A$ with $A-N$ separable (where $N$ is the nil radical of $A$ ) for which there is no subalgebra $S$ such that $S=A-N$ and that this is the case even if only algebras over fields $K$ of characteristic not 2 are considered. However there are important subclasses of the class of noncommutative Jordan algebras for which the result does hold. Early work was done for the cases of the Jordan and the alternative algebras [4], [9], [13], motivated by the corresponding result for the associative algebras [2]. More recently, some activity has been directed towards obtaining as large as possible a class of noncommutative Jordan algebras for which this structure theorem holds [12], [14].

A possible direction of investigation stems from the observation that in the

Received by the editors May 29, 1973.

AMS (MOS) subject classifications (1970). Primary 17A20, 17A15; Secondary 17A05, $17 \mathrm{~A} 30$.

Key words and phrases. Wedderburn decomposition, flexible algebras, noncommutative Jordan algebra, quasiassociative algebra, power-associative al gebra.

(1) This paper contains results from the author's $\mathrm{Ph}$. D. dissertation written at the University of Missouri at Columbia under the guidance of Professor D. J. Rodabaugh. 
known counterexamples, $A-N$ is simple and of degree not exceeding 2. For example, it follows readily from the work of McCrimmon [6] that the conclusion of the structure theorem holds whenever $A-N$ has its simple summands each of degree exceeding 2. Similar results have been obtained by Hemminger for the case of the commutative algebras [5]. It is the result of the present paper that this type of result also holds for the case of the strictly power-associative flexible algebras. The results of this paper generalize much of [5]. In what follows, all algebras are assumed to be finite dimensional.

In [10], Rodabaugh has identified sufficient conditions on a class of algebras for each member of the class to have a Wedderburn decomposition. We will take advantage of these results in order to obtain our basic working lemma.

Definition 1. A class $P$ of algebras will be called a decomposable class if for each algebra $A$ in the class $P$ :

(a) $A$ is strictly power-associative over a field of characteristic not 2 or 3.

(b) $A-N$ is in $P$.

(c) If $B$ is a subalgebra of $A$ whose image in $A \rightarrow A-N$ is a nonnil ideal in $A-N$, then $B$ is in $P$.

(d) A semisimple implies $A=A_{1} \oplus A_{2} \oplus \cdots \oplus A_{t}$ where each $A_{i}$ is simple with an identity element.

(e) $A(e, t) A(e, t) \subseteq A(e, t)(t=0,1)$ if $e$ is an idempotent and where $A(e, t)$ is the usual Peirce subspace corresponding to $e$ and $t$.

It has been pointed out that the restriction away from characteristic 3 in [10] can be removed. The class $\mathcal{G}$ resp. $\mathcal{F}$ of noncommutative Jordan algebras resp. strictly power-associative, flexible algebras over a field of characteristic not 2 or 3 and such that, $A-N$ is separable and the simple summands of $A-N$ have at least 3 pairwise orthogonal idempotents each, is a decomposable class.

Here, an algebra is flexible if it satisfies the identity

$$
(x, y, x)=0
$$

or equivalently when char $K \neq 2$

$$
F(x, y, z)=(x, y, z)+(z, y, x)=0 .
$$

A flexible algebra is a noncommutative Jordan algebra if it satisfies the additional identity

$$
\left(x^{2}, y, x\right)=0 .
$$

If char $K \neq 2,3$ this is equivalent to the linearized form

$$
J(x, y, z, w)=(x, y, z \cdot w)+(z, y, w \cdot x)+(w, y, x \cdot z)=0 .
$$

Here $a \cdot b=(a b+b a) / 2$. The algebra obtained from $A$ by redefining multiplication 
in this manner is called $A^{+}$. An algebra is strictly power-associative if every scalar extension satisfies the identity $x^{1} x^{k}=x^{1+k}$ for all positive integers 1 and $k$. One consequence of power-associativity when char $K \neq 2$ is

$$
\begin{aligned}
E(x, y, z, w)= & J(x, y, z, w)+J(y, z, w, x) \\
& +J(z, w, x, y)+J(w, x, y, z)=0 .
\end{aligned}
$$

The necessary results needed to show that the classes $\mathcal{G}$ and $\mathcal{F}$ are decomposable classes are contained in [1], [8] and [10].

A power-associative algebra $A$ will be called nearly-simple if it has no ideals distinct from $0, N$, and $A$.

Lemma 1. If $P$ is a decomposable class of algebras sucb that, if $A$ is in $P$ and if $M \subseteq N$ is an ideal of $A$ then $A-M$ is in $P$, then every member of $P$ bas a Wedderburn decomposition provided that every nearly-simple algebra with identity element and contained in $P$ bas a Wedderburn decomposition.

Proof. By Theorem 2.1 of [10] it is sufficient to demonstrate the result for each member $A$ of $P$ such that $A-N$ is simple and $A$ has an identity element. An algebra of minimum dimension in a decomposable class must be semisimple and hence have a Wedderburn decomposition. This provides the initial step for an induction argument on the dimension of the algebra with Lemma 2.2 of [10] providing the induction step.

It is noted that both the classes $\mathcal{G}$ and $\mathcal{F}$ have the property that if $M \subseteq N$ is an ideal of an algebra $A$ in the class then $A-M$ is in the class. Because of this it is then fruitful to consider the nearly-simple algebras in these classes.

Basic results on flexible algebras are found in [1] and [8]. Below are recalled a few of these which are used most heavily here.

If $A$ is a nonnil power-associative algebra then $A$ has an idempotent $e$ and relative to $e$, the Peirce decomposition $A=A(e, 0)+A(e, 1 / 2)+A(e, 1)$ where $A(e, i)=\{x$ in $A: x e+e x=2 i x\}$. For $x$ in $A, x=x_{1}+x_{1 / 2}+x_{0}$ where $x_{i}$ is in $A(e, i)$. For $S$ a subspace of $A$, we shall write $S(e, i)=\left\{x_{i}: x\right.$ is in $\left.S\right\}$. Occasionally $S(e, i)$ will be denoted by $S_{i}$. For convenience here, if $S$ and $T$ are subspaces then $S \odot T$ will denote $S T+T S$. If $A$ is a flexible algebra then $A(e, 0)$ and $A(e, 1)$ are subalgebras and $A$ has the multiplication properties $A(e, 0) \odot$ $A(e, 1)=0, A(e, i) \odot A(e, 1 / 2) \subseteq A(e, 1 / 2)+A(e, 1-i)$ where $i$ is 0 or 1 . If $x$ is in $A(e, 1 / 2)$ then $e x$ and $x e$ are in $A(e, 1 / 2)$.

When a flexible algebra $A$ has $n$ pairwise orthogonal idempotents such that $1=\sum_{i=1}^{n} e_{i}$ then $A$ has a decomposition $A=\Sigma A_{i j}$ where $1 \leq i, j \leq n, A_{i i}=$ $A\left(e_{i}, 1\right)$ and $A_{i j}=A_{j i}=A\left(e_{i}, 1 / 2\right) \cap A\left(e_{j}, 1 / 2\right)$. The subspaces $A_{i i}$ are subalgebras and if $i, j, k$ and $m$ denote pairwise distinct subscripts then 


$$
\begin{gathered}
A_{i i} A_{j j}=0, \quad A_{i i} \odot A_{i j} \subseteq A_{i j}+A_{j j}, \quad A_{i i} \odot A_{j k}=0, \quad A_{i j}^{2} \subseteq A_{i i}+A_{i j}+A_{j j} \\
A_{i j} \odot A_{j k} \subseteq A_{i k}, \quad A_{i j} A_{k m}=0, \quad \text { and } A_{i j} \cdot A_{i j} \subseteq A_{i i}+A_{j i}
\end{gathered}
$$

When $A$ is decomposed relative to $n$ pairwise orthogonal idempotents then the component of $x$ in the subspace $A_{i j}$ will be denoted by $x_{i j}$ and the notation $B_{i j}=\left\{x_{i j}: x\right.$ is in $\left.B\right\}$ will be adopted for subspaces $B$ of $A$.

For a noncommutative Jordan algebra with identity $1=\sum_{i=1}^{n} e_{i}$ where the $e_{i}$ are pairwise orthogonal idempotents and $n \geq 2$, the corresponding Peirce decomposition is said to be interconnected if $\left[A_{i j} \cdot A_{i j}\right]_{i i}=A_{i i}$ for all $i \neq j[6$, p. 12]. $\mathrm{McC}$ rimmon has shown that any Peirce decomposition of an arbitrary simple noncommutative Jordan algebra is interconnected [6, p. 14]. This result holds also for nearly-simple noncommutative Jordan algebras and essentially the same proof can be used. The ideal $\left[A_{1 / 2}^{2}\right]_{0}+A_{1 / 2}+\left[A_{1 / 2}^{2}\right]_{1}$ cannot be $N$ when $e \neq 1$ since then $A_{1 / 2} \subseteq N$ and $A-N$ is the direct sum of two nonzero ideals contrary to fact. Thus this ideal is either 0 or $A$ which is the same conclusion obtained if $A$ is simple.

It is then a corollary to Lemma 7 of [6] that a nearly-simple algebra with $1=$ $\sum_{i=1}^{n} e_{i}$ for $n \geq 3$ has a regular indicator and by Theorem 4 of [6] is then either a commutative Jordan algebra of characteristic not 2 or is a quasiassociative algebra. For these two classes of algebras, a Wedderburn principal theorem is known. The first class is considered in [4] and [9] while the quasiassociative algebras are a special case of the algebras considered in [14, Satz 11].

The following sequence of lemmas will show that in the case of the class $\mathcal{F}$ of strictly power-associative flexible algebras that the nearly-simple algebras with identity either have a Wedderburn decomposition or are noncommutative Jordan or have degree less than 3. It will be assumed in the following that $A$ is a strictly power-associative flexible algebra over a field of characteristic not 2 or 3 with $A-N$ separable and with at least 3 pairwise orthogonal idempotents.

Relative to an idempotent $e$ there are subspaces $C(e)=\{x$ in $A(e, 1): x$. $A(e, 1 / 2)=0\}$ and $B(e)=\{x$ in $A(e, 1): x \cdot A(e, 1 / 2) \subseteq A(e, 0)\}$. Clearly $C(e) \subseteq B(e)$ for any idempotent $e$. Oehmke has shown [8] that if $e$ is an idempotent of $A$ distinct from 1 , then $C(e)$ is an ideal of $A, B(e)$ is an ideal of $A(e, 1)$ and that $C(e) A(e, 1 / 2)=A(e, 1 / 2) C(e)=0$. It is also known from [8] that the simple, strictly power-associative, flexible algebras over a field of characteristic not 2 or 3 are either Jordan or quasiassociative. It is this consideration which motivates the first of the following results.

Lemma 2. If $A-N$ is commutative and $N \subseteq A(e, 0)+A(e, 1)$ for an idempotent $e$ in $A$ then $x y=y x$ for every $x$ and $y$ in $A(e, 1 / 2)$.

Proof. When $x$ is in $A(e, 1 / 2)$ then $e x-x e$ is in $A(e, 1 / 2)$. Since $A-N$ is 
commutative, $e x-x e$ is also in $N$ and hence $e x=x e$. This with $e x+x e=x$ implies $e x=x e=(1 / 2) x$ for every $x$ in $A(e, 1 / 2)$. Now if $x$ and $y$ are in $A(e, 1 / 2)$ then for $\lambda=0$ or $1,0=[F(y, x, e)]_{\lambda}=(\lambda-1 / 2)[y x-x y]_{\lambda}$ and hence $[y x-x y]_{\lambda}=0$. Since $A-N$ is commutative, $[y x-x y]_{1 / 2}$ is in $N$ and is hence 0 . Thus $y x=x y$.

Lemma 3. If $A-N$ is associative and $N \subseteq A(e, 0)+A(e, 1)$ for an idempotent $e$ in $A$, then $\left[A(e, 1 / 2)^{2}\right]_{\lambda} \subseteq[A(e, 1 / 2) \cdot A(e, 1 / 2)]_{\lambda}$ for $\lambda=0$ or 1 .

Proof. Let $x$ be an element of $A(e, 1 / 2)$. Then $(e, e, x)=(e, x, e)=(x, e, e)$ $=0$ since these associators are in both $A(e, 1 / 2)$ and in $N$. Let $L=\{e x: x$ is in $A(e, 1 / 2)\}$ and $R=\{x e: x$ is in $A(e, 1 / 2)\}$. Since $x=e x+x e$ for $x$ in $A(e, 1 / 2)$ then $A(e, 1 / 2)=L+R$. From $(e, e, x)=0$ comes the fact $e(e x)=e x$. Similarly $(x e) e=$ $x e$. Then $(e x) e=e(x e)=e(e x)+e(x e)-e x=e(e x+x e-x)=0$. Hence $e x=0$ and $x e=x$ for each $x$ in $R$ while $e x=x$ and $x e=0$ for each $x$ in $L$. If $x$ is in $R \cap L$ then $x=e x=0$ so $A(e, 1 / 2)$ is a vector space direct sum of $R$ and $L$.

Now if $x$ is in $A$ then $[x]_{1 / 2}=4 x \cdot e-4(x \cdot e) \cdot e$ is known from [1]. Now let $a$ and $b$ be in $A(e, 1 / 2)$. Then if $\bar{x}$ denotes the image of $x$ in $A-N$ then we have $[\bar{a} \bar{b}]_{1 / 2}$ $=\overline{0}$. Thus $\overline{0}=4(\bar{a} \bar{b}) \cdot \bar{e}-4((\bar{a} \bar{b}) \cdot \bar{e}) \cdot \bar{e}=\overline{[a b]_{1 / 2}}$ so that $[a b]_{1 / 2}$ is in $N$ and is hence 0 . Therefore $A(e, 1 / 2)^{2} \subseteq A(e, 0)+A(e, 1)$.

Let $x$ be in $R$ and $y$ be in $L$. Then, for $i=0$ or $1,0=[F(x, y, e)]_{i}=i[x y]_{i}$ $+(1-i)[y x]_{i}$. Hence $x y$ is in $A(e, 0)$ and $y x$ is in $A(e, 1)$. That is $R L \subseteq$ $A(e, 0)$ and $L R \subseteq A(e, 1)$. However if $x$ and $y$ are both in $R$ then for $i=0$ or $1,0=[F(x, y, e)]_{i}=(i-1)[x y]_{i}-i[y x]_{i}$ which implies $R^{2}=0$. Similarly $L^{2}=0$.

Now let $x=a+b$ and $y=c+d$ where $a$ and $c$ are in $R$ and $b$ and $d$ are in $L$. Then $[x y]_{i}=[a c+a d+b c+b d]_{i}=(1-i)[a d]_{i}+i[b c]_{i}=(1-i)[a \cdot d]_{i}+$ $i[b \cdot c]_{i}$ so that $\left[A(e, 1 / 2)^{2}\right]_{i}$ is contained in $[A(e, 1 / 2) \cdot A(e, 1 / 2)]_{i}$ for $i=0$ or 1 which is the claim of the lemma.

If $A$ has pairwise orthogonal idempotents $e$ and $f$ then $C(e)$ and $C(f)$ are ideals of $A$ such that $C(e) \cap C(f)=0$. When $A$ is nearly-simple this implies that either $C(e)$ or $C(f)$ is 0 . Also if $1=e_{1}+e_{2}+e_{3}$ and $i, j$, and $k$ are pairwise distinct then $C\left(e_{i}\right) \subseteq C\left(e_{i}+e_{j}\right)$ since $x \cdot A\left(e_{i}+e_{j}, 1 / 2\right) \subseteq x \cdot A_{i k}+x \cdot A_{j k}$ $=0$ where $x$ is in $C\left(e_{i}\right)$. These facts together imply that there is no loss of generality in assuming that $C\left(e_{3}\right)=0$ when $A$ is nearly-simple and also that $C\left(e_{i}+e_{2}\right)$ is either $N$ or 0 .

The role of the sequence of Lemmas $4-12$ is to reduce the problem to the case in which $C\left(e_{1}+e_{2}\right)=0$. The procedure is to assume that $C\left(e_{1}+e_{2}\right)=N$ which after intervening results will yield $N=0$.

Let $A$ be a strictly power-associative, flexible algebra with $A-N$ separable, $A$ nearly-simple over a field of characteristic not 2 or 3 and $1=e_{1}+e_{2}$ $+e_{3}$. Since $A-N$ is then simple it is either commutative or quasiassociative. 
An algebra is quasiassociative if there is an extension field $K$ of $F$ so that the algebra $A_{K}(\lambda)$ is associative for some choice of $\lambda$ in $K, \lambda \neq 1 / 2$, by redefining multiplication as $x * y=\lambda x y+(1-\lambda) y x$.

The algebras $A$ and $A(\lambda)$ are identical with respect to subspaces, subalgebras, and ideals. Further, since powers in $A$ and $A(\lambda)$ coincide, $N(\lambda)$ is the nil radical of $A(\lambda)$. Also the algebras $A^{+}$and $A(\lambda)^{+}$coincide so that $A(e, i)=$ $[A(\lambda)](e, i)$ for $i=0,1 / 2,1$ and $x \cdot y=(x y+y x) / 2=(x * y+y * x) / 2$.

In what follows, $K$ will denote the algebraic closure of $F$. This is a sufficiently large field so that when $A-N$ is quasiassociative then there is an element $\lambda$ in $K$ such that $(A-N)(\lambda)$ is associative. Since $A-N$ is separable, $N_{K}$ is the nil radical of $A_{K}$. Reference to $A$ and to $N$ will now be as algebras over $K$ except where otherwise noted. As an algebra over $K, A$ has all the properties mentioned above that it has as an algebra over $F$, except that $A$ is perhaps not nearly-simple as an algebra over $K$.

If $e$ is an idempotent of $A$ such that $N \subseteq A(e, 0)+A(e, 1)$ then since $A-N$ is either commutative or quasiassociative it follows from Lemmas 2,3 that either $\left[A(e, 1 / 2)^{2}\right]_{\lambda} \subseteq[A(e, 1 / 2) \cdot A(e, 1 / 2)]_{\lambda}$ for $\lambda=0$ and 1 or else $[A(e, 1 / 2) * A(e, 1 / 2)]_{\lambda} \subseteq$ $[A(e, 1 / 2) \cdot A(e, 1 / 2)]_{\lambda}$. But $A(e, 1 / 2)^{2} \subseteq A(e, 1 / 2) * A(e, 1 / 2)$ since for algebras $A, A(\mu)$ $=B$ if and only if $B\left(\mu^{\prime}\right)=A$ for some $\mu^{\prime}$ whenever $\mu \neq 1 / 2$. Thus in any case $\left[A(e, 1 / 2)^{2}\right]_{\lambda} \subseteq[A(e, 1 / 2) \cdot A(e, 1 / 2)]_{\lambda}$ for $\lambda=0$ and 1 .

The next two lemmas establish identities which shall be major tools in the development.

Lemma 4. For any idempotent $e$, if $a$ is in $A(e, 1)$ and $b$ and $c$ are in $A(e, 1 / 2)$ then $(c \cdot b) a=c e(b \cdot a)+b e(a \cdot c)+e c(b \cdot a)+e b(c \cdot a)-(1 / 2) c(a b)-$ $(1 / 2) b(a c)$.

Proof. Applying the facts $(e, a, b \cdot c)=a(b \cdot c)_{1}+a(b \cdot c)_{0}-e\left[a(b \cdot c)_{1}\right]-$ $e\left[a(b \cdot c)_{0}\right]=a(b \cdot c)_{1}-e\left[a(b \cdot c)_{1}\right]=0,(a, e, b \cdot c)=0$, and that $x e+e x=x$ for each $x$ in $A(e, 1 / 2)$ to $E(a, b, c, e)-(1 / 2) F(a, c, b)-(1 / 2) F(a, b, c)-(1 / 2) F(b, a, c)$ $=0$ yields this identity.

Lemma 5. If $a$ is in $A(e, 0)$ and $b$ and $c$ are in $A(e, 1 / 2)$ then $a(b \cdot c)=$ $(1 / 2)(a b) c+(1 / 2)(a c) b+b(c \cdot a)+c(a \cdot b)-b[e(c \cdot a)]-c[e(a \cdot b)]-e[b(c \cdot a)]-$ $e[c(b \cdot a)]$.

Proof. This follows as in the previous lemmas by expanding $E(a, b, c, e)-$ $(1 / 2) F(b, a, c)=0$ and using the facts that $(a, e, b \cdot c)=(e, a, b \cdot c)=0$ and that $e x+x e=x$ for each $x$ in $A(e, 1 / 2)$.

Lemma 6. If $N \subseteq A\left(e_{k}+e_{j}, 1\right)+A\left(e_{k}+e_{j}, 0\right)$ then $\left[A_{i k}^{2}\right]_{k k} \subseteq\left[A_{i k} \cdot A_{i k}\right]_{k k}$. If $N \subseteq A_{11}+A_{22}+A_{33}$ then $\left[A_{i k}^{2}\right]_{k k} \subseteq\left[A_{i k} \cdot A_{i k}\right]_{k k}$ for all choices of $i$ and $k$ distinct. 
Proof. Let $e=e_{k}+e_{j}$. Then $\left[A(e, 1 / 2)^{2}\right]_{1} \subseteq[A(e, 1 / 2) \cdot A(e, 1 / 2)]_{1}$ where the 1 denotes the component in $A(e, 1)$. The properties of subspace multiplication along with $A(e, 1 / 2)=A_{i k}+A_{i j}$ give $\left[A_{i k}^{2}+A_{i k} A_{i j}+A_{i j} A_{i k}+A_{i j}^{2}\right]_{1}$ contained in $\left[A_{i k} \cdot A_{i k}+A_{i k} \cdot A_{i j}+A_{i j} \cdot A_{i j}\right]_{1}$. Then taking the components of each of these in $A_{k k}$ gives $\left[A_{i k}^{2}\right]_{k k} \subseteq\left[A_{i k} \cdot A_{i k}\right]_{k k}$. If $N$ is in $A_{11}+A_{22}+A_{33}$ then the above hypothesis is satisfied for all choices of $i$ and $k$.

Lemma 7. If $\left[A_{i k}^{2}\right]_{k k} \subseteq\left[A_{i k} \cdot A_{i k}\right]_{k k}$ then $\left[A_{i k}^{2}\right]_{k k} \odot A_{j k} \subseteq A_{j k}$ for $i, j$ and $k$ pairwise distinct.

Proof. Let $a$ be in $A_{j k}$ and $b$ and $c$ in $A_{i k}$. If $e=e_{j}+e_{k}$ then $a$ is in $A(e, 1)$ and $b$ and $c$ are in $A(e, 1 / 2)$ so by Lemma 4 and the fact $A_{i j} A_{j k} \subseteq A_{i k}$ it follows that $(c \cdot b) a$ is in $A_{j k}$. Then $\left[A_{i k}^{2}\right]_{k k} A_{j k} \subseteq\left[A_{i k} \cdot A_{i k}\right]_{k k} A_{j k} \subseteq\left(A_{i k} \cdot A_{i k}\right) A_{j k}$ $\subseteq A_{j k}$. By the flexibility of $A, a(c \cdot b)=(c \cdot b) a+(a c) b+(a b) c-b(c a)-c(b a)$ which is in $A_{j k}$ and hence $A_{j k}\left[A_{i k}^{2}\right]_{k k} \subseteq A_{j k}$.

A flexible algebra $A$ is called stable relative to an idempotent $e$ if $A(e, \lambda)$ $\odot A(e, 1 / 2) \subseteq A(e, 1 / 2)$ for $\lambda=0$ and 1 . If $A-N$ is stable then $a b$ and $b a$ are in $A(e, 1 / 2)+N$ whenever $a$ is in $A(e, \lambda)$ and $b$ is in $A(e, 1 / 2)$ where $\lambda$ is 0 or 1 . For an idempotent $e$ the subspace $H(e)$ of $A$ is defined by $H(e)=A(e, 1 / 2)+$ $\left[A(e, 1 / 2)^{2}\right]_{0}+\left[A(e, 1 / 2)^{2}\right]_{1}$.

Lemma 8. The subspace $H(e)+N$ is an ideal of $A$ for any idempotent $e$ of A. This bolds in both $A_{F}$ and $A_{K}$.

Proof. The algebra $A-N$ is noncommutative Jordan since it is either commutative Jordan or quasiassociative. Under the natural homomorphism from $A$ to $A-N$ the preimage of the subspace $H(\bar{e})$ is $H(e)+N$. It is known [7] that $H(\bar{e})$ is an ideal of $A-N$ so $H(e)+N$ is an ideal of $A$.

Since $A_{F}$ is nearly-simple, we are concerned with the consequences in $A_{F}$ of the supposition $N=C(f)$ where $f=e_{1}+e_{2}$. The property $N \odot A(f, 1 / 2)=0$ holds, even in $A_{K}$, since the products involved depend only on the multiplication of basis elements for these subspaces.

Lemma 9. If $N \odot A(f, 1 / 2)=0$ where $f=e_{1}+e_{2}$ then the subspace $I(f)=$ $[A(f, 0) \odot A(f, 1 / 2)]_{1}$ is a nil ideal of $A$ and is contained in $A\left(e_{1}, 1\right)+A\left(e_{2}, 1\right)$. This bolds in both $A_{K}$ and $A_{F}$.

Proof. Let $x$ be in $A(f, 0), y$ in $A(f, 1)$, and $w$ in $A(f, 1 / 2)$. Then

$$
\begin{aligned}
0 & =[E(x, y, w, e)-(1 / 2) F(y, x, w)]_{1} \\
& =[(1 / 2)(w x) y-(1 / 2) x(y w)+(x w) y-x(w y)-x(e(y \cdot w))-x(y \cdot w)]_{1} .
\end{aligned}
$$

Thus $[(1 / 2)(w x) y+(x w) y]_{1}$ is in $I(f)$. The identities (2) and (5) hold in $A^{+}$so the above argument can also be used to obtain $[(3,2)(w \cdot x) \cdot y]_{1}$ in $I(f)$. In light of 
our restrictions on characteristic this gives $[(w \cdot x) \cdot y]_{1}$ in $I(f)$. Then

$$
\begin{aligned}
{[(x w) y]_{1}=} & 2[(1 / 2)(w x) y+(x w) y]_{1}-4[(w \cdot x) \cdot y]_{1} \\
& -[F(w, x, y)+F(x, w, y)]_{1}+[x(w y)-(y w) x]_{1}
\end{aligned}
$$

is in $I(f)$. Then $[(w x) y]_{1}=2\left[(1 / 2)(w x) y+(x w)_{y}\right]_{1}$ is in $I(f)$ as are $[y(x w)]_{1}=$ $[(w x) y]_{1}-[F(w, x, y)]_{1}$ and $[y(w x)]_{1}=[(x w) y]_{1}-[F(x, w, y)]_{1}$. Thus $I(f)$ is an ideal of $A(f, 1)$.

When $x$ is in $A(f, 0)$ and $y$ is in $A(f, 1 / 2)$ then $(x y)_{1}$ is in $N$ since $A-N$ is noncommutative Jordan and hence stable. Similarly, $(y x)_{1}$ is in $N$ so that $I(f) \subseteq N$. Since $N \odot A(f, 1 / 2)=0$ and $A(f, 0) \odot A(f, 1)=0$ then $l(f)$ is a nil ideal of $A$. Finally,

$$
\begin{aligned}
I(f) & =\left[A_{33} \odot\left(A_{13}+A_{23}\right)\right](f, 1) \subseteq\left[A_{13}+A_{11}+A_{23}+A_{22}\right](f, 1) \\
& =A_{11}+A_{22} .
\end{aligned}
$$

Lemma 10. If $f=e_{1}+e_{2}$ satisfies $N=C(f)$, then the algebras $A_{F}$ and $A_{K}$ are both stable relative to $f$.

Proof. First consider $A_{F}$. Again by the stability of $A-N, A(f, 1) \odot$ $A(f, 1 / 2)$ is contained in $A(f, 1 / 2)+N$. But since $A$ is a flexible algebra, $A(f, 1) \odot$ $A(f, 1 / 2) \subseteq A(f, 1 / 2)+A(f, 0)$. But $N$ is contained in $A(f, 1)$ so $A(f, 1) \odot A(f, 1 / 2)$ is in $A(f, 1 / 2)$. Now $I(f)$ is a nil ideal contained in $A_{11}+A_{22}$ and if $I(f)=0$ then $A$ is stable relative to $f$ so assume that $I(f)=N$. Let $g=e_{k}+e_{3}$ where $k=1$ or 2. Then $H(g)+N$ is an ideal of $A$. Now $H(g)+N$ is not 0 or $N$ for if $H(g)+$ $N \subseteq N$ then $A(g, 0)+N$ and $A(g, 1)+N$ are distinct proper ideals of $A$ contrary to the near-simplicity of $A$. Thus $H(g)+N=A$. If $I(f)=N$ and $H(g)+N=A$ when $A$ is considered as an algebra over $F$ then they hold when $A$ is considered as an algebra over $K$.

Now consider $A$ as an algebra over $K$. It follows that if $j=3-k$ then $A_{33}=$ $[H(g)+N]_{33} \subseteq\left[A(g, 1 / 2)^{2}\right]_{33}=\left[\left(A_{j k}+A_{j 3}\right)^{2}\right]_{33}=\left[A_{j 3}^{2}\right]_{33}$. Since $I(f)=N$ is in $A_{11}+$ $A_{22}$ the Lemmas 5 and 6 hold for any choice of $i, j$, and $k$. Then

$$
\begin{aligned}
A(f, 0) \odot A(f, 1 / 2) & =A_{33} \odot\left[A_{13}+A_{23}\right] \subseteq A_{33} \odot A_{13}+A_{33} \odot A_{23} \\
& \subseteq\left[A_{23}^{2}\right]_{33} \odot A_{13}+\left[A_{13}^{2}\right]_{33} \odot A_{23} \subseteq A_{13}+A_{23}=A(f, 1 / 2)
\end{aligned}
$$

and $A_{K}$ is stable relative to $/$. Then $A_{F}$ is stable relative to $f$.

Lemma 11. If $A$ is stable relative to an idempotent $e$ then $H(e)$ is an ideal of $A$.

Proof. By virtue of the stability of $A$ it is sufficient to show that $A(e, \lambda) \odot$ 
[A(e, 1/2) $]_{\lambda} \subseteq H(e)$ for $\lambda=0$ and 1. Let $a$ be an element of $A(e, 1)$ and let $b$ and $c$ be in $A(e, 1 / 2)$. Then by flexibility and Lemma $4, a(c \cdot b)=(c \cdot b) a-c(b a)-b(c a)$ $+(a b) c+(a c) b=c[e(b \cdot a)]+b[e(a \cdot c)]+e[c(b \cdot a)]+e[b(c \cdot a)]-(1 / 2) c(a b)-$ $(1 / 2) b(a c)-c(b a)-b(c a)+(a b) c+(a c) b$. The component of the right member in $A(e, 1)$ is in $\left[A(e, 1 / 2)^{2}\right]_{1}$ since $A$ is stable relative to $e$. Then since $\left[A(e, 1 / 2)^{2}\right]_{1}$ $\subseteq[A(e, 1 / 2) \cdot A(e, 1 / 2)]_{1}$ it follows that

$$
A(e, 1)\left[A(e, 1 / 2)^{2}\right]_{1} \subseteq A(e, 1)\left[A(e, 1 / 2) \cdot A(e, 1 / 2]_{1} \subseteq\left[A(e, 1 / 2)^{2}\right]_{1} \subseteq H(e) .\right.
$$

Similar work with flexibility and Lemmas 4,5 , and 6 yields the remaining three necessary containments.

Lemma 12. The subspace $C(f)$ in $A_{F}$ with $f=e_{1}+e_{2}$ is the zero subspace.

Proof. As remarked earlier, $C(f)$ is either $N$ or 0 so assume $C(f)=N$. Then the algebra $A_{K}$ is stable relative to $f$ so $H(f)$ is an ideal of $A_{K}$. Then $H(f) \cap$ $A_{F}$ is an ideal of $A_{F}$. The ideal $H(f) \cap A_{F}$ contains $A_{F}(f, 1 / 2)$ and hence by the near-simplicity of $A_{F}$, cannot be 0 or $N_{F}$ since then $A_{F}(f, 0)+N$ and $A_{F}(f, 1)+$ $N$ are distinct proper ideals of $A_{F}$. Thus $H(f) \cap A_{F}=A_{F}$ and hence $H(f)=A$. This places $f$ in the subspace $\left[A(f, 1 / 2)^{2}\right]_{1} \subseteq[A(f, 1 / 2) \cdot A(f, 1 / 2)]_{1}$. Let $n$ be in $N$ and $f=\left[\sum_{s=1}^{m} a_{s} \cdot b_{s}\right]_{1}$ where $a_{s}$ and $b_{s}$ are in $A(f, 1 / 2)$ for $s=1, \ldots, m$. Then for each $s$,

$$
\begin{aligned}
{\left[a_{s} \cdot b_{s}\right]_{1} n=\left(a_{s} \cdot b_{s}\right) n=} & a_{s}\left(n b_{s}\right)+b_{s}\left[f\left(a_{s} \cdot n\right)\right]+f\left[a_{s}\left(b_{s} \cdot n\right)\right] \\
& +f\left[b_{s}\left(a_{s} \cdot n\right)\right]-(1 / 2) a_{s}\left(n b_{s}\right)-(1 / 2) b_{s}\left(n a_{s}\right)
\end{aligned}
$$

by Lemma 5. The right member is 0 since $N \odot A(e, 1 / 2)=0$. Then since $n$ is in $A(f, 1), n=f n=\left[\sum_{s=1}^{m}\left(a_{s} \cdot b_{s}\right)\right]_{1} n=0$ and hence $C(f)=N=0$.

It follows from the preceding series of lemmas and relabeling that in $A_{F}$, $C(f)=C(g)=C(b)=0$ where $f=e_{1}+e_{2}, g=e_{1}+e_{3}$, and $b=e_{2}+e_{3}$. In [8, p. 224] Oehmke uses this result (the result follows there from an assumption of simplicity which we have avoided here) to show that $B=B\left(e_{1}\right)+B\left(e_{2}\right)+B\left(e_{3}\right)$ is an ideal of $A$. He also establishes that if $B=0$ then $A$ is a noncommutative Jordan algebra. These algebras were discussed in the first portion of this paper. Assume then that $B \neq 0$. Also, $B \neq A$ since if $B=A$ then $A \subseteq A_{11}+A_{22}+A_{33}$ and $A$ is a direct sum of three proper ideals contrary to its near-simplicity. With this in mind we assume $B=N$ and thus that $N \subseteq A_{11}+A_{22}+A_{33}$. This condition holds in $A_{K}$ as well as in $A_{F}$. As a result of this $N \subseteq A(e, 1)+A(e, 0)$ where $e$ is $f, g$, or $b$ and the results of Lemmas $2,3,6,7$, and 8 hold. These conditions are now sufficient to show that $H(f)+N$ is a Wedderburn decomposition of $A$. When $A$ is decomposed relative to a set of $n$ pairwise orthogonal idempotents, $B \circ C$ shall denote $\Sigma_{i j}[B \odot C]_{i j}$ where $i$ and $j$ range between and include 1 
and $n$. By $B^{(2)}$ we shall mean $B \circ B$. In the following lemma, $f$ shall denote $e_{i}+e_{j}$

Lemma 13. If $i, j$, and $k$ are pairwise distinct then $\left[A_{i j}^{2}\right]_{i i}=\left[A_{i k}^{2}\right]_{i i}$.

Proof. As before, $A=H(f)+N$ and $A(f, 1 / 2)=A_{i k}+A_{j k}$. Since $N \cap A_{i j}=0$, $A_{i j} \subseteq\left(A_{i k}+A_{j k}\right)^{(2)} \subseteq A_{i k}^{(2)}+A_{i k} \odot A_{j k}+A_{j k}^{(2)}$. But $A_{i j} \cap A_{i k}^{(2)}=A_{i j} \cap A_{j k}^{(2)}=0$ so $A_{i j} \subseteq A_{i k} \odot A_{j k}$. Now let $a$ be in $A_{i k}, b$ in $A_{k j}$, and $c$ be in $A_{i j}$. By Lemma 5 ,

$$
(a \cdot b) c=a[f(b \cdot c)]+b[f(a \cdot c)]+f[a(b \cdot c)]+f[b(a \cdot c)]-(1 / 2) a(c b)-(1 / 2) b(c a) .
$$

The left member is contained in $A(f, 1)$ and hence equals the component of the right member in $A(f, 1)$; that is,

$$
(a \cdot b)_{c}=\{a[f(b \cdot c)]+b[f(a \cdot c)]+(1 / 2) a(b c)+(1 / 2) b(a c)\}_{1} .
$$

The components in $A_{i i}$ of the members of this equation are then equal which yields $\left[(a \cdot b)_{c}\right]_{i i}=\{a[f(b \cdot c)]+(1 / 2) a(b c)\}_{i i}$ which is contained in $\left[A_{i k}^{2}\right]_{i i}$. Therefore

$$
\left[A_{i j}^{2}\right]_{i i}=\left[\left(A_{i k} \odot A_{k j}\right) A_{i j}\right]_{i i} \subseteq\left[\left(A_{i k} \cdot A_{j k}\right) A_{i j}\right]_{i i} \subseteq\left[A_{i k}^{2}\right]_{i i}
$$

Here the containment $A_{i k} A_{k j} \subseteq A_{i k} \cdot A_{j k}$ follows from $\left[A(f, 1 / 2)^{2}\right]_{1} \subseteq$ $[A(f, 1 / 2) \cdot A(f, 1 / 2)]_{1}$ and $\left[\left(A_{i k}+A_{j k}\right)^{2}\right]_{i j}=\left[A_{i k} \odot A_{j k}\right]_{i j}$. By relabeling this also proves the reverse inclusion.

Lemma 14. The subspaces $A_{i j}$ and $A_{i k} A_{j k}$ are identical.

Proof. Since $A$ is flexible, $A_{i j} \supseteq A_{i k} \odot A_{j k}=A_{i k} \circ A_{k j}$. The reverse inclusion was shown in the first part of Lemma 13.

Lemma 15. The subspace $\left[A_{i k}^{2} \odot A_{j k}^{2}\right]_{k k}$ is contained in the subspace $\left[A_{i k}^{2}+A_{j k}^{2}\right]_{k k}$.

Proof. Let $x$ and $y$ be in $A_{i k}$ and let $z$ and $w$ be in $A_{j k}$. By power-associativity $E(x, y, z, w)=0$. Expanding and collecting terms yields

$$
\begin{aligned}
4(x \cdot y) \cdot(z \cdot w)= & x[y(z \cdot w)]+y[x(z \cdot w)]+z[w(x \cdot y)]+w[z(x \cdot y)]-(z, y, w \cdot x) \\
& -(w, y, x \cdot z)-(y, z, w \cdot x)-(x, z, y \cdot w) \\
& -(x, w, y \cdot z)-(y, w, z \cdot x)-(w, x, y \cdot z)-(z, x, w \cdot y) .
\end{aligned}
$$

It was shown in the proof of Lemma 7 that $A_{j k}\left[A_{i k} \cdot A_{i k}\right] \subseteq A_{j k}$ for $i, j$, and $k$ pairwise distinct and hence the right member of the above equality is contained in $A_{i j}^{2}+A_{j k}^{2}+A_{i k}^{2}$. The component of $(x \cdot y) \cdot(z \cdot w)$ in the subspace $A_{k k}$ is thus contained in $\left[A_{j k}^{2}+A_{i k}^{2}\right]_{k k}$. Then $(x y)_{k k} \cdot(z w)_{k k}$ is in $\left(A_{i k} \cdot A_{i k}\right)_{k k} \cdot\left(A_{j k} \cdot A_{j k}\right)_{k k}$ (by Lemma 6 ) and hence in $\left[A_{i k}^{2}+A_{j k}^{2}\right]_{k k}$. By applying the flexible law, 


$$
\begin{aligned}
(x y)_{k k}(z w)_{k k}-(z w)_{k k}(y x)_{k k} & =[(x y)(z w)-(z w)(y x)]_{k k}=\left\{x[y(z w)]-[(z w) y]_{x}\right\}_{k k} \\
& =\left\{x\left[y(z w)_{k k}\right]-\left[(z w)_{k k} y\right]_{k k}\right.
\end{aligned}
$$

which is in $\left\{A_{i k}\left[A_{i k}\left(A_{j k} \cdot A_{j k}\right)_{k k}\right]+\left[\left(A_{j k} \cdot A_{j k}\right)_{k k} A_{i k}\right] A_{i k}\right\}_{k k}$ this in turn is contained in $\left[A_{i k}^{2}\right]_{k k}$ and hence in $\left[A_{j k}^{2}+A_{i k}^{2}\right]_{k k}$. Subtracting $(x y)_{k k}(z w)_{k k}-$ $(z w)_{k k}(y x)_{k k}$ from twice $(x y)_{k k} \cdot(z w)_{k k}$ gives $(z w)_{k k}(y \cdot x)_{k k}$ in $\left[A_{j k}^{2}+A_{i k}^{2}\right]_{k k}$ and hence $\left[A_{j k}^{2} A_{i k}^{2}\right]_{k k} \subseteq\left[A_{j k}^{2}\right]_{k k}\left[A_{i k} \cdot A_{i k}\right]_{k k} \subseteq\left[A_{j k}^{2}+A_{i k}^{2}\right]_{k k} \cdot$ A completely similar argument shows that $\left[A_{i k}^{2} A_{j k}^{2}\right]_{k k}$ is contained in $\left[A_{j k}^{2}+A_{i k}^{2}\right]_{k k}$.

Lemma 16. The subspace $A_{i k} A_{j k}^{(2)}+A_{j k}^{(2)} A_{i k}$ is contained in the subspace $A_{i j}+A_{i k}$

Proof. By Lemmas 14 and $7, A_{i k} A_{j k}^{(2)}=A_{i k}\left[A_{j k}^{2}\right]_{j j}+A_{i k}\left[A_{j k}^{2}\right]_{j k}+A_{i k}\left[A_{j k}^{2}\right]_{k k}$ $\subseteq A_{i j}+A_{i k}$. Similarly $A_{j k}^{(2)} A_{i k}$ is contained in $A_{i j}+A_{i k}$.

Lemma 17. The subspace $A_{i k}^{(2)} A_{i k}^{(2)}$ is contained in the subspace $A_{i k}+A_{i k}^{(2)}$.

Proof. Lemmas 7, 13, 14, and 15 yield

$$
\begin{aligned}
A_{i k}^{(2)} A_{i k}^{(2)} & =\left(\left[A_{i k}^{2}\right]_{i i}+\left[A_{i k}^{2}\right]_{i k}+\left[A_{i k}^{2}\right]_{k k}\right)^{2} \\
& \subseteq\left[A_{i k}^{2}\right]_{i i}\left[A_{i k}^{2}\right]_{i i}+\left[A_{i k}^{2}\right]_{i i} \odot A_{i k}+A_{i k}^{2}+A_{i k} \odot\left[A_{i k}^{2}\right]_{k k}+\left[A_{i k}^{2}\right]_{k k}\left[A_{i k}^{2}\right]_{k k} \\
& \subseteq\left[A_{i k}^{2}\right]_{i i}\left[A_{i j}\right]_{i i}+\left[A_{i j}^{2}\right]_{i i} \odot A_{i k}+A_{i k}^{2}+A_{i k} \odot\left[A_{j k}^{2}\right]_{k k}+\left[A_{j k}^{2}\right]_{k k}\left[A_{i k}^{2}\right]_{k k} \\
& \subseteq\left[A_{i k}^{2}\right]_{i i}+\left[A_{i j}^{2}\right]_{i i}+A_{i k}+A_{i k}^{2}+\left[A_{j k}^{2}\right]_{k k}+\left[A_{i k}^{2}\right]_{k k} \\
& =\left[A_{i k}^{2}\right]_{i i}+A_{i k}+A_{i k}^{2}+\left[A_{i k}^{2}\right]_{k k} \subseteq A_{i k}^{(2)}+A_{i k} .
\end{aligned}
$$

Lemma 18. The subspace $A_{i k} \cdot A_{i k}^{(2)}$ is contained in the subspace $A_{i k}+A_{i k}^{2}$.

Proof. By Lemmas 7 and 13, $A_{i k}\left(\left[A_{i k}^{2}\right]_{i i}+\left[A_{i k}^{2}\right]_{i k}+\left[A_{i k}^{2}\right]_{k k}\right)=A_{i k}\left[A_{i k}^{2}\right]_{i i}+$ $A_{i k}\left[A_{i k}^{2}\right]_{i k}+A_{i k}\left[A_{i k}^{2}\right]_{k k} \subseteq A_{i k}\left[A_{i j}^{2}\right]_{i i}+A_{i k}^{2}+A_{i k}\left[A_{j k}^{2}\right]_{k k} \subseteq A_{i k}+A_{i k}^{2}$. Similarly $A_{i k}^{(2)} A_{i k}$ is contained in $A_{i k}+A_{i k}^{2}$.

Lemma 19. The subspace $A_{i k}^{(2)} \odot A_{j k}^{(2)}$ is contained in the subspace $A_{i j}+$ $A_{i k}+A_{i k}+\left[A_{i k}^{2}+A_{j k}^{2}\right]_{k k}$.

Proof. By Lemmas 7 and 15,

$$
\begin{aligned}
A_{i k}^{(2)} A_{j k}^{(2)} & =\left[A_{i k}^{2}\right]_{i k}\left[A_{j k}^{2}\right]_{j k}+\left[A_{i k}^{2}\right]_{i k}\left[A_{j k}^{2}\right]_{k k}+\left[A_{i k}^{2}\right]_{k k}\left[A_{j k}^{2}\right]_{j k}+\left[A_{i k}^{2}\right]_{k k}\left[A_{j k}^{2}\right]_{k k} \\
& \subseteq A_{i k} A_{j k}+A_{i k}\left[A_{j k}^{2}\right]_{k k}+\left[A_{i k}^{2}\right]_{k k} A_{j k}+\left[A_{i k}^{2}+A_{j k}^{2}\right]_{k k} \\
& \subseteq A_{i j}+A_{i k}+A_{j k}+\left[A_{i k}^{2}+A_{j k}^{2}\right]_{k k}
\end{aligned}
$$

Interchanging $i$ and $j$ gives the containment for $A_{j k}^{(2)} A_{i k}^{(2)}$. 
Lemma 20. The subspace $H(f)$ is a subalgebra of $A_{K}$ wbere $f=e_{1}+e_{2}$.

Proof. The subspace $H(f)=A(f, 1 / 2)=A(f, 1 / 2)^{(2)}$ can be written in terms of the subspaces $A_{i j}$ as

$$
A_{13}+A_{23}+\left[A_{13}+A_{23}\right]^{(2)}=A_{13}+A_{23}+A_{13}^{(2)}+A_{12}+A_{23}^{(2)}
$$

(by Lemma 14). Now $\left(A_{13}+A_{23}\right)\left(A_{13}+A_{23}\right) \subseteq A(f, 1 / 2)^{(2)}$. By Lemmas 16 and 18,

$$
\left(A_{13}+A_{23}\right) \odot\left[A_{13}^{(2)}+A_{12}+A_{23}^{(2)}\right] \subseteq A_{13}+A_{13}^{2}+A_{23}+A_{12}+A_{23}^{2} .
$$

Finally

$$
\begin{aligned}
{\left[A_{13}^{(2)}+A_{12}\right.} & \left.+A_{23}^{(2)}\right]\left[A_{13}^{(2)}+A_{12}+A_{23}^{2}\right] \\
& \subseteq A_{13}+A_{13}^{(2)}+A_{12}+A_{23}+\left[A_{13}^{2}\right]_{33}+\left[A_{23}^{2}\right]_{33}+A_{12}^{2}+A_{23}^{2} \\
& \subseteq A_{13}+A_{23}+A_{12}+A_{13}^{(2)}+A_{23}^{(2)}+A_{12}^{2}
\end{aligned}
$$

by Lemmas $16,17,18$, and 19. But $A_{12}^{2} \subseteq A_{12}^{(2)}=\left[A_{12}^{2}\right]_{11}+\left[A_{12}^{2}\right]_{12}+\left[A_{12}^{2}\right]_{22} \subseteq\left[A_{13}^{2}\right]_{11}$ $+A_{12}+\left[A_{23}^{2}\right]_{22} \subseteq H(f)$. Thus $H(f)$ is a subalgebra of $A_{K}$.

Lemma 21. The algebra $A_{F}$ is a vector space direct sum of its nil radical $N$ and the subalgebra $H(f) \cap A_{F}$.

Proof. By Lemma 10, $H(f)+N$ is an ideal of $A_{K}$ and hence $[H(f)+N] \cap A_{F}$ is an ideal of $A_{F}$. Since $A_{F}$ is nearly-simple, $A_{F}(f, 1 / 2) \subseteq H(f)$ is not contained in $N$ and thus $A_{F}=[H(f)+N] \cap A_{F}$. Therefore $H(f)+N=A_{K}$ and $A_{F}=$ $\left[H(f) \cap A_{F}\right]+N_{F}$.

Now let $x$ be in $N_{F} \cap\left[H(f) \cap A_{F}\right]$. Then since in $A_{F}, N \subseteq A_{11}+A_{22}+A_{33}$, then $x=x_{1}+x_{2}+x_{3}$ where $x_{i}$ is in $A_{i i}$. Each $x_{i}$ is also contained in the corresponding subspace of $A_{K}$. Now in $A_{K}$ it follows from Lemmas 13 and 14 that $[H(f)]_{i i}=\left[A(f, 1 / 2)^{2}\right]_{i i}=\left[A_{13}^{2}+A_{12}+A_{23}^{2}\right]_{i i}=\left[A_{13}^{2}+A_{23}^{2}\right]_{i i} \subseteq\left[A_{i j}^{2}\right]_{i i}$ for each $i \neq j$. Since $x_{i}$ is in $[H(f)]_{i i}$, then by Lemma $7, x_{i} \cdot A(e, 1 / 2)=x_{i} \cdot\left[A_{i j}+A_{i k}\right]=$ $x_{i} \cdot A_{i j}+x_{i} \cdot A_{i k}$ is contained in $A_{i j}+A_{i k}$. Also since $x_{i}$ is in $N_{F} \subseteq N_{K}$ then $x_{i} \cdot A(e, 1 / 2)$ is contained in $A_{11}+A_{22}+A_{33}$. Hence $x_{i} \cdot A(e, 1 / 2)=0$ for $i=1$, 2, and 3. Therefore in $A_{F}, x_{i}$ is in $C\left(e_{i}\right)=0$ for $i=1,2$, and 3 and thus $\left[H(f) \cap A_{F}\right] \cap N_{F}=0$.

The results of the sequence of Lemmas 2-21 may be condensed into the following theorem.

Theorem 1. Let $A$ be a nearly-simple, strictly power-associative, flexible algebra with unity over a field of characteristic not 2 or 3 , with $1=e_{1}+e_{2}+e_{3}$, and with $A-N$ separable. Then $A=S+N$ where $S$ is a subalgebra of $A$. 
Then in light of Lemma 1 we have the following Wedderburn principal theorem for flexible algebras.

Theorem 2. Let $A$ be a strictly power-associative, flexible algebra over a field $F$ with characteristic not 2 or 3 , with $A-N$ separable and such that $A=$ $A_{1} \oplus A_{2} \oplus \cdots \oplus A_{n}$ where each $A_{i}$ bas $A_{i}-N_{i}$ simple and has more than two pairwise ortbogonal idempotents. Then $A=S+N$ where $S$ is a subalgebra of $A$.

\section{BIBLIOGRAPHY}

1. A. A. Albert, Power-associative rings, Trans. Amer. Math. Soc. 64 (1948), 552593. MR 10, 349.

2. - Structure of algebras, Amer. Math. Soc. Colloq. Publ., vol. 24, Amer. Math. Soc., Providence, R. I., 1961. MR 23 \#A912.

3. - A theory of power-associative commutative algebras, Trans. Amer. Math. Soc. 69 (1950), 503-527. MR 12, 475.

4. A. A. Albert, The Wedderbum principal theorem for Jordan algebras, Ann. of Math. (2) 48 (1947), 1-7. MR 8, 435.

5. R. L. Hemminger, On the Wedderburn principal theorem for commutative powerassociative algebras, Trans. Amer. Math. Soc. 121 (1966), 36-51. MR 34 \#2642. $1-33$.

6. K. McCrimmon, Non commutative Jordan rings, Trans. Amer. Math. Soc. 158 (1971),

7. - Structure and representations of noncommutative Jordan algebras, Trans. Amer. Math. Soc. 121 (1966), 187-199. MR 32 \#5700.

8. R. H. Oehmke, On flexible algebras, Ann. of Math. (2) 68 (1958), 221-230. MR 21 $\# 5664$.

9. A. J. Penico, The Wedderburn principal theorem for Jordan algebras, Trans. Amer. Math. Soc. 70 (1951), 404-420. MR 12, 798.

10. D. J. Rodabaugh, On the Wedderburn principal theorem, Trans. Amer. Math. Soc. 138 (1969), 343-361.

11. R. D. Schafer, An introduction to nonassociative algebras, Pure and Appl. Math., vol. 22, Academic Press, New York, 1966. MR 35 \#1643.

12. - On generalized standard algebras, Proc. Nat. Acad. Sci. U.S.A. 60 (1968), 73-74. MR $39 \# 4238$.

13. - The Wedderbum principal theorem for alternative algebras, Bull. Amer. Math. Soc. 55 (1949), 604-614. MR 10,676.

14. A. Thedy, Zum Wedderbumshen Zerlegungssatz, Math. Z. 113 (1970), 173-195. MR $41 \# 8486$.

DEPARTMENT OF MATHEMATICS, CENTRAL MICHIGAN UNIVERSITY, MT. PLEASANT, MICHIGAN 48858 